\title{
Interfering with miR-24 alleviates rotenone-induced do- paminergic neuron injury via enhancing autophagy by up-regulating DJ-1
}

\author{
Biao $\mathrm{Ge}^{\mathrm{a}}$, Hairong $\mathrm{Wu}^{\mathrm{b}}$, Dan Shao ${ }^{\mathrm{b}}$, Shulin $\mathrm{Li}^{\mathrm{b}}$, Fengrui $\mathrm{Li}^{\mathrm{b}}{ }^{\text {** }}$ \\ ${ }^{a}$ Department of Geriatrics, Baogang Hospital of Inner Mongolia, Baotou; 014010, China. \\ ${ }^{b}$ College of Basic Medicine and Forensic Medicine, Baotou Medical College, Baotou; 014060, China.
}

\begin{abstract}
Background: To investigate the mechanism of miR-24 on dopaminergic neuron injury in parkinson's disease (PD).

Methods: In vivo and in vitro PD model was established using rotenone. All rats were received behavioral assessments using open field test and rotarod test. MN9D cell viability was measured using MTT assay. The relationship between miR-24 and DJ-1 was detected using dual-luciferase reporter assay.

Results: MiR-24 expression was increased in the brain tissues from PD rats and rotenone-induced MN9D cells, while DJ-1 expression, the LC3-II/LC3-I ratio and the protein expression of Beclin 1 was decreased. Rotenone reduced MN9D cell viability, but interfering with miR-24 changed the inhibition effect of rotenone. Moreover, interfering with miR-24 enhanced the LC3-II/LC3-I ratio and the protein expression of Beclin 1 by increasing DJ-1 expression, and then relieved rotenone-induced dopaminergic neuronal cell injury. These findings were also proved in PD rat model.

Conclusion: Interfering with miR-24 alleviated rotenone-induced dopaminergic neuron injury via enhancing autophagy by increasing DJ-1.
\end{abstract}

Keywords: Parkinson's disease, autophagy, miR-24, DJ-1, dopaminergic neuron

\section{Introduction}

As the second most common neurodegenerative disease, the incidence of Parkinson's disease (PD) in elderly patients is $2 \%$ and can reach $5 \%$ in people over 85 years old [1]. The main pathological changes of PD are necrosis of dopaminergic neurons in the compact part of the substantia nigra ( $\mathrm{SN}$ ) and the formation of Lewy bodies [2]. The pathogenesis of PD is complicated and not clearly understood. Recently, it has been reported that autophagy is involved in PD [3]. Autophagy is widespread in eukaryotic cells and is a pathway for lysosomes to degrade their own organelles and other macromolecules. Autophagy dysfunction has been implicated in the pathogenesis of many neurodegenerative disorders, including PD [4].

\footnotetext{
* Corresponding author: Fengrui Li

Mailing address: College of Basic Medicine and Forensic Medicine, Baotou Medical College, 31 Jianshe Rd, Baotou 014060, People's Republic of China.

Email: rui6826@qq.com
}

Received: 18 December 2019 / Accepted: 20 December 2019
DJ-1 is a member of the peptidase C56 family encoded by the PARK7 gene, a PD-related pathogenic gene, which can protect neurons from oxidative stress and cell death [5]. DJ-1 deficiency is related to the development of PD [6]. De Miranda et al. [7] reported that overexpression of DJ-1 protected against rotenone-induced neurotoxicity in a rat model of PD. In addition, it has been reported that DJ-1 can act as a regulator of autoph PD. Xu et al. [8] found that knockdown of DJ-1 aggravated $\alpha$-synuclein aggregation by inhibiting the activation of chaperone-mediated autophagy and then promoted the development of PD. Although many studies have investigated the role of DJ-1 in PD, the molecular mechanism of DJ-1 in autophagy in PD is not completely understood.

MicroRNAs (miRNAs) are a class of noncoding RNAs with a length between 18 and 25 nucleotides, which play an important role in various cellular biology processes [9]. Recently, growing evidence has revealed that multiple miRNAs are involved in the development of PD. For example, serum miR-221 can act as a PD biomarker [10], and miR-30e can regulate neuroinflammation in PD by targeting Nlrp3 ers [13]. In an expression profile study, miR24 was found to be highly expressed in PD [14]. However, its role in PD has not been reported. In addition, miR-24 is 
known to be related to autophagy. Tong et al. [15] found that overexpression of miR-24 induced autophagy by increasing the level of sirtuin 1 (SIRT1) in uterine sarcoma. However, the relationship between miR-24 and autophagy in PD is not clear. Bioinformatics analysis with TargetScan shows that miR-24 has binding sites with DJ-1. Therefore, we speculated that the role of miR-24 in autophagy in PD might be related to DJ-1.

Rotenone, a plant-derived insecticide, can cause specific injury to dopaminergic neurons and induce Parkinson's disease-like symptoms [16]. In this study, we investigated the mechanism of miR-24 in autophagy in PD using rotenone-induced injury of dopaminergic neurons in rats and cultured cells, aiming to provide a possible target for the prevention and treatment of PD.

\section{Methods}

\section{Animals}

Twenty-four adult Wistar rats (200-250 g) were purchased from Beijing Vital River Laboratory Animal Technology Co., Ltd. (Beijing, China). All rats were housed under a 12/12-h light/dark cycle at $25 \pm 2{ }^{\circ} \mathrm{C}$. The study was approved by the Ethics Committee of Baotou Medical College.

\section{PD rat model}

The expressions of miR-152, MKK7 mRNA, and MKK7 mRNA in cells were detected using quantitative real-time PCR (qRT-PCR). Total RNAs were extracted from the tumor tissues and cells using Trizol reagent (Invitrogen, Waltham, MA, US). The TaqMan Reverse Transcription Kit (Applied Biosystems, Foster City, CA, US) was used to reversely transcribe RNAs into cDNAs. The qRT-PCR was conducted using the SYBR Premix Ex TaqTM II Kit (Takara, Dalian, Liaoning, China) on an ABI 7500 RealTime PCR system (Applied Biosystems, Waltham, MA, US). U6 was used as the internal control for miR-152, and GAPDH was used as the internal control for mRNAs. The relative expressions were calculated using the $2^{-\Delta \Delta \mathrm{Ct}}$ method.

\section{Cell transfection}

The PD rat model was established as previously described [17]. Briefly, rats received one subcutaneous injection of rotenone (in a dose of $1.5 \mathrm{mg} / \mathrm{kg}$ dissolved in dimethyl sulfoxide [DMSO]) every other day for a total of 21 days.

\section{Animal grouping}

The rats were divided into 4 groups of 6 rats: (1) Sham group: rats received one subcutaneous injection of DMSO $(0.1 \mathrm{~mL} / 100 \mathrm{~g})$ every other day; (2) PD group: the model group; (3) miR-24 inhibitor group: rats were anesthetized by intraperitoneal injection of $6 \%$ chloral hydrate $(6 \mathrm{~mL} /$ $\mathrm{kg})$. An adenovirus vector-mediated miR-24 inhibitor (5 $\mu \mathrm{L}$; Genechem, Shanghai, China) was injected into the SN of rats at the following stereotactic coordinates: $-5.2 \mathrm{~mm}$ from the bregma, $2.0 \mathrm{~mm}$ to the left of the midline, and 7.5 mm below the subdural matter [18]; (4) negative control (NC) group: rats were anesthetized by intraperitoneal injection of $6 \%$ chloral hydrate $(6 \mathrm{~mL} / \mathrm{kg})$. Negative control of adenovirus vector-mediated miR-24 inhibitor $(5 \mu \mathrm{L}$; Genechem) was injected into the SN of rats at the same site. After 4 weeks of injections, the rats in the miR-24 inhibitor group and the NC group were injected with rotenone in a dose of $1.5 \mathrm{mg} / \mathrm{kg}$ at the same site. At the end of the experiments, all rats underwent behavioral assessment using an open field test and a rotarod test. They were subsequently euthanized, and their brains were obtained for further experiments.

\section{Tyrosine hydroxylase (TH) staining}

TH staining was used to evaluate the histopathological changes in rats. Briefly, tissue sections were pre-incubated for $30 \mathrm{~min}$ in 5\% normal bovine serum and then incubated with diluted TH antibody (abcam, Cambridge, UK) overnight at $4{ }^{\circ} \mathrm{C}$. The next day, the corresponding biotinylated secondary antibody was added and incubated for 1-2 h, then added avidin/biotin reagent (Dako, High Wycombe, UK) to continue incubation, and stained with diaminobenzidine- $\mathrm{HCl}(\mathrm{DAB})$.

\section{Open field test}

The open field test was performed as previously described [18]. A square wooden box $(80 \times 80 \times 40 \mathrm{~cm})$ with red walls and a white smooth polished floor divided by black lines into 16 equal squares of $4 \times 4 \mathrm{~cm}$ was used. The rats were placed in the central area of the open field and allowed to freely explore it for $3 \mathrm{~min}$. A video camera was fixed to the top of the box to record their movement and behavior for off-line analysis. Behavioral changes (i.e., latency, rearing, ambulation, and immobility time) were recorded.

\section{Rotarod test}

The rotarod test was performed using a rotary rod apparatus as previously described [18]. The rats were placed in the apparatus (diameter: $3 \mathrm{~cm}$, height: $90 \mathrm{~cm}$ ), which was operated at a constant speed of $10 \mathrm{rpm}$. Before the experiment, the rats were pre-trained for 3 days. After the open field test, the animals were evaluated for a period of $5 \mathrm{~min}$, and the time spent by each rat on the rod was recorded.

\section{Cell culture}

A dopaminergic neuronal cell line (MN9D cells) was purchased from American Type Culture Collection (Manassas, VA, USA). The cells were maintained in Dulbecco's Modified Eagle Medium (DMEM; Gibco, Carlsbad, CA, USA) supplemented with $10 \%$ fetal bovine serum (Gibco), $100 \mathrm{U} / \mathrm{mL}$ penicillin (Invitrogen, Waltham, MA, USA), and $100-\mu \mathrm{g} / \mathrm{mL}$ streptomycin (Invitrogen) and cultured in an incubator with a humidified atmosphere of $95 \%$ air and $5 \% \mathrm{CO}_{2}$ at $37^{\circ} \mathrm{C}$. 


\section{Cell treatment}

MN9D cells were treated with rotenone to establish the in vitro PD model. The cells were exposed to different concentrations of rotenone $(10,20,50$, and $100 \mathrm{nM})$ for $24 \mathrm{~h}$. For cell transfection, they were transfected with miR-24 inhibitor, miR-24 mimic, si-DJ-1, pcDNA-DJ-1, or their negative control using Lipofectamine 2000 (Invitrogen) for $24 \mathrm{~h}$ prior to $100 \mathrm{nM}$ rotenone exposure.

\section{MTT assay}

MN9D cell viability was measured using a 3-(4,5-dimethylthiazol-2-yl)-2,5-diphenyltetrazolium bromide (MTT) assay. The cells were seeded into 96-well plates at a density of $1 \times 10^{4}$ cells/well and cultured for $24 \mathrm{~h}$. They were then transfected with miR-24 inhibitor and/or si-DJ-1 for $24 \mathrm{~h}$, followed by exposure to $100 \mathrm{nM}$ rotenone for another $24 \mathrm{~h}$. Next, the old medium was removed, and the MTT solution $(0.5 \mathrm{mg} / \mathrm{mL}, 10 \mu \mathrm{L})$ was added into each well. Four hours later, the supernatants were removed, and $150 \mu \mathrm{L}$ of DMSO was added into each well to dissolve the resultant formazan crystals. The optical density value was read at $490 \mathrm{~nm}$ using a microplate reader. Results are expressed as the percentage of MTT reduction, assuming the absorbance of the control cells to be $100 \%$.

\section{Quantitative real-time polymerase chain reaction}

Total RNA was extracted from tissues or cells using TRIzol reagent (Invitrogen). Then, complementary DNA (cDNA) was synthesized using a PrimeScript One Step RT-PCR Kit (Takara Biotechnology, Dalian, China) following the manufacturer's protocol. Quantitative real-time polymerase chain reaction (qRT-PCR) was performed with an ABI PRISM 5700 Sequence Detection System (Applied Biosystems, Foster City, CA, USA). The relative expression of miR-24, miR-30e, miR-29b-3p, miR-652, miR-1274, and DJ-1 mRNA was calculated using the 2 ${ }^{\Delta \Delta \mathrm{Ct}}$ method.

\section{Western blot}

Total protein was extracted from tissues and cells using radioimmunoprecipitation assay (RIPA) buffer. Then, an equal amount of total protein $(10 \mu \mathrm{g})$ was separated by $12 \%$ sodium dodecyl sulfate-polyacrylamide gel electrophoresis (SDS-PAGE) and transferred onto polyvinylidene fluoride (PVDF) membranes (MilliporeSigma, Burlington, MA, USA). The membranes were first blocked with $5 \%$ skim milk at room temperature for $1 \mathrm{~h}$ and then incubated with the primary antibodies overnight at $4{ }^{\circ} \mathrm{C}$. After being washed three times with tris-buffered saline with tween (TBST), the membranes were incubated with the corresponding horseradish peroxidase (HRP)-conjugated secondary antibodies at room temperature for $1 \mathrm{~h}$. The immune-blot signals were developed using an enhanced chemiluminescence method. The anti-DJ-1 antibody, anti-LC3-I antibody, anti-LC3-II antibody, anti-Beclin 1 antibody, and anti- $\beta$-actin antibody (Cell Signaling Technology, Danvers, MA, USA) were used as the primary antibodies at 1:1000 dilutions.

\section{Dual-luciferase reporter assay}

DJ-1 three prime untranslated region (3'UTR) fragments containing wild type (WT) or mutant (Mut) miR24 binding sites were amplified and cloned by PCR. The PCR products were inserted into a pMIR-REPORT-DJ1-3'UTR plasmid (Invitrogen). MN9D cells were seeded into a 24-well plate at a density of $1 \times 10^{5}$ cells $/ \mathrm{mL}$ and cultured for $24 \mathrm{~h}$. They were then transfected with a WT or Mut pMIR-DJ-1-3'UTR plasmid, along with miR-24 mimic or inhibitor using Lipofectamine 2000 (Invitrogen). Forty-eight hours later, the relative luciferase activity was analyzed using a Dual-Luciferase Reporter Assay System (Promega, Madison, WI, USA).

\section{Statistical analysis}

All data were presented as means \pm standard deviations and processed with GraphPad Prism 7.0. The difference between the two groups was analyzed using a Student's $t$-test. The difference among more than two groups was analyzed by a one-way analysis of variance (ANOVA), followed by the Newman-Keuls post hoc test. A $P$-value of $<0.05$ was considered statistically significant.

\section{Results}

\section{MiR-24 expression increased in the brain tissues of PD rats}

To investigate the relationship between miR-24 and PD, rats were divided into the Sham group and PD group. From the results of Tyrosine hydroxylase (TH) staining, compared with the Sham group, the number of TH-positive neurons in the PD group was significantly reduced. In addition, rats in the PD group had a significant deterioration in motor performance and coordination (Figure 1A). Then, we conducted a screening experiment for miRNAs. From the qRT-PCR results, we found that miR-24 expression was upregulated while miR-30e expression was downregulated in the PD group (Figure 1B). The expressions of miR-29b-3p, miR-652, and miR-1274 had no significant change in the brain tissues of $\mathrm{PD}$ rats compared with the Sham group (Figure 1B). The mechanism of miR-30e in PD has been reported [11]. but the mechanism of miR-24 in PD has not been reported. For this reason, we selected miR-24 for further study. We also measured the expressions of DJ-1, LC3-I, LC3-II, and Beclin 1 in the brain tissues of these rats. Compared with the Sham group, DJ-1 expression, the LC3-II/LC3-I ratio, and the protein level of Beclin 1 were downregulated in the brain tissues of PD rats (Figure 1C).

\section{Interfering with miR-24 alleviated rotenone-induced dopaminergic neuronal cell injury}

To investigate whether miR-24 played an important role in rotenone-induced dopaminergic neuron injury, we first 


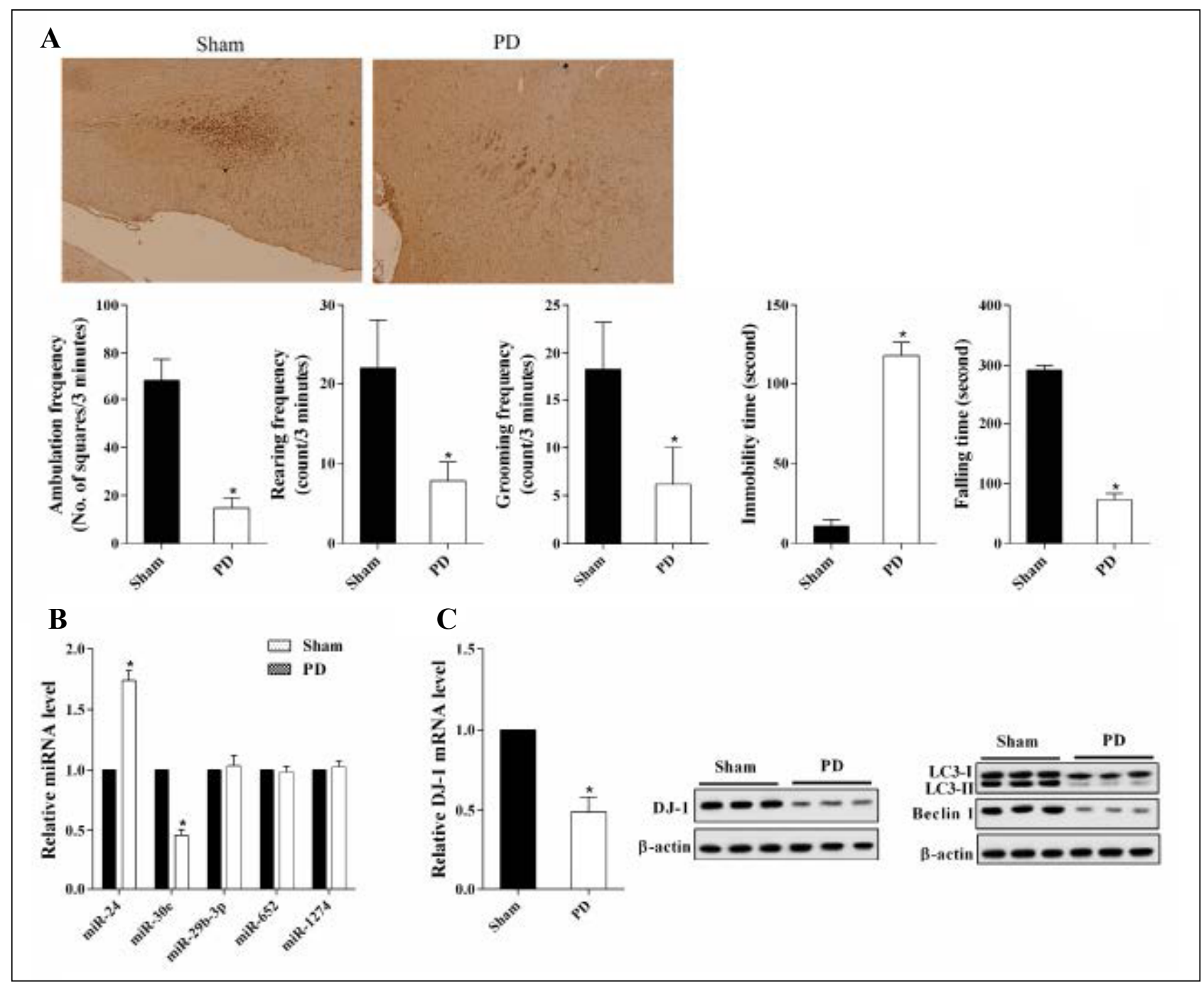

Figure 1. MiR-24 expression in the brain tissues of Parkinson's disease (PD) rats. Rats were divided into a Sham group and a PD group of 6 rats each. (A) Tyrosine hydroxylase (TH) staining, Open field test, and rotarod test. $* P<0.05$, vs Sham. (B) The expressions of miR-24, miR-30e, miR29b-3p, miR-652 and miR-1274 in the brain tissues of these rats. (C) The expressions of DJ-1, LC3-I, LC3-II, and Beclin 1 in the brain tissues of these rats. $* P<0.05$, vs Sham.

investigated its expression in MN9D cells treated with different concentrations of rotenone $(10,20,50$, and 100 $\mathrm{nM})$. As shown in Figure 2A, miR-24 expression was dose-dependently up-regulated after rotenone treatment, while DJ-1 expression was dose-dependently downregulated. Thus, we chose $100 \mathrm{nM}$ of rotenone for further experiments. We divided the MN9D cells into Control, Rotenone, Rotenone $+\mathrm{NC}$, and Rotenone + miR-24 inhibitor groups. The results showed that the miR-24 inhibitor offset the up-regulation of miR-24 expression and the down-regulation of DJ-1 expression induced by rotenone (Figure 2B, C). The results of the MTT assay showed that interfering with miR-24 changed the inhibition effect of rotenone on MN9D cell viability (Figure 2D).

MiR-24 affected autophagy by targeting DJ-1 and rotenone affected dopaminergic neuronal cell injury through miR-24/DJ-1

To explore the mechanism of miR-24, we tried to investigate its target gene. Based on bioinformatics analysis with
TargetScan, we found that DJ-1 was one of the putative target genes of miR-24 (Figure 3A). From the results of dual-luciferase reporter assay, miR-24 could negatively regulate the luciferase activity of DJ-1 (Figure 3B). To test whether miR-24 regulated DJ-1 expression, we measured DJ-1 expression upon the transfection of miR-24 inhibitor or mimic. The results of the qRT-PCR analysis suggested that the mRNA and protein levels of DJ-1 were significantly up-regulated after the transfection of miR24 inhibitor, while the transfection of miR-24 mimic produced the opposite effect (Figure 3C). Besides, to investigate the role of miR-24/DJ-1 in autophagy, we initially measured the protein levels of LC3-I, LC3-II, and Beclin 1 in MN9D cells treated with rotenone, miR-24 inhibitor, si-DJ-1, and RAPA (autophagy agonist). The results showed that miR-24 inhibitor offset the down-regulation of LC3-II/LC3-I ratio and Beclin 1 protein levels induced by rotenone, while si-DJ-1 could reverse the effect of miR-24 inhibitor (Figure 3D). As expected, RAPA, in turn, reversed the effect of si-DJ-1 (Figure 3D). Then, we 


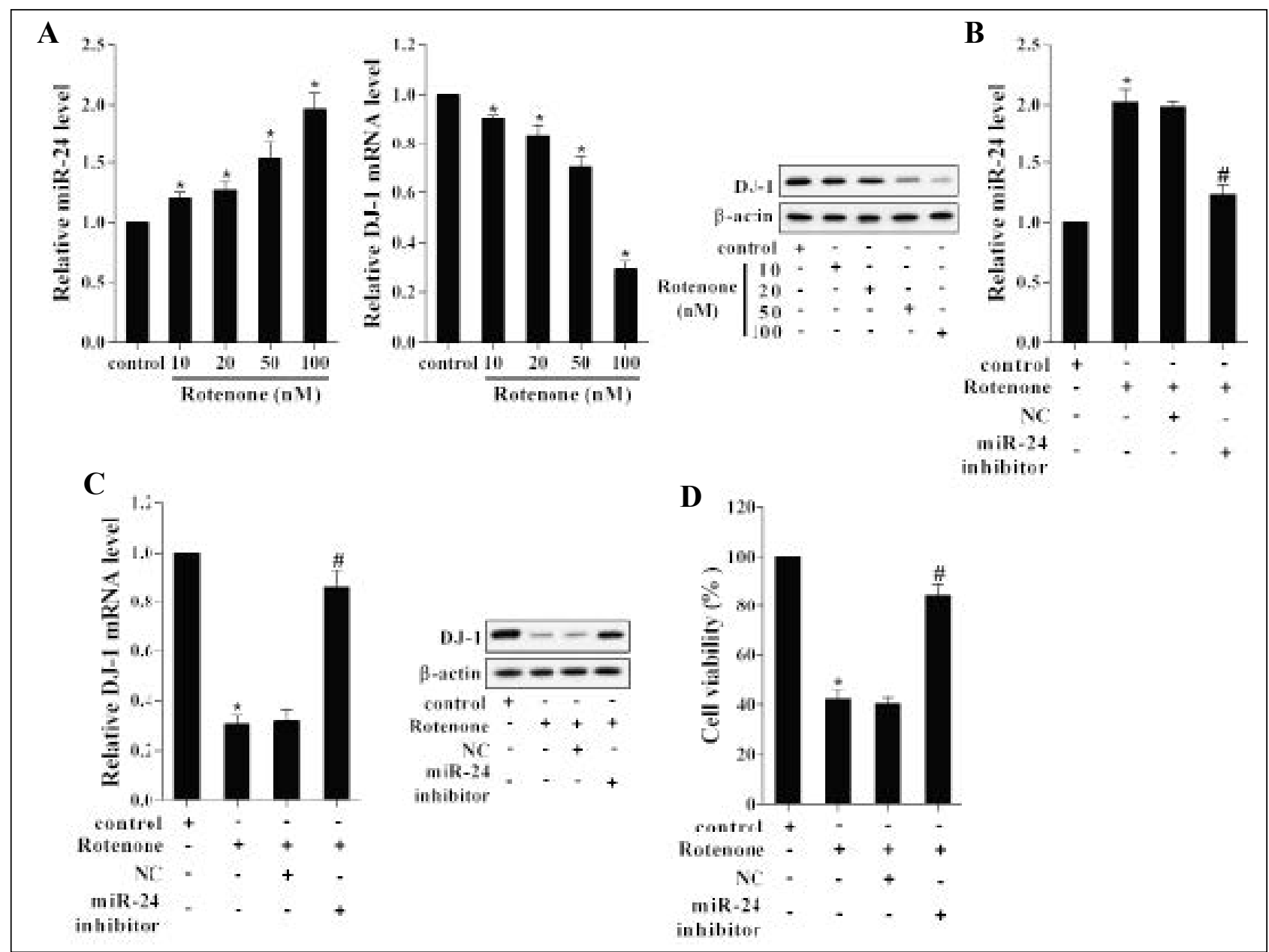

Figure 2. Interfering with miR-24 alleviated rotenone-induced dopaminergic neuronal cell injury. MN9D cells were treated with different concentrations of rotenone (10, 20, 50, and $100 \mathrm{nM}$ ). (A) The expressions of miR-24 and DJ-1. MN9D cells were divided into Control, Rotenone, Rotenone + negative control $(\mathrm{NC})$, and Rotenone + miR-24 inhibitor groups. $* P<0.05$, vs control. (B) MiR-24 expression. $* P<0.05$, vs control. $\# P<0.05$, vs Rotenone + NC. (C) DJ-1 expression. * $P<0.05$, vs control. $\# P<0.05$, vs Rotenone + NC. (D) MN9D cell viability. $* P<0.05$, vs control. \# $P<$ 0.05 , vs Rotenone + NC.

detected the protein levels of LC3-I, LC3-II, and Beclin 1 in MN9D cells treated with miR-24 mimic, pcDNA-DJ-1, and 3-MA (autophagy inhibitor). As shown in Figure $3 \mathrm{E}$, the overexpression of miR-24 could down-regulate the LC3-II/LC3-I ratio and Beclin 1 protein level, while the transfection of pcDNA-DJ-1 changed this trend. As expected, 3-MA, in turn, reversed the effect of pcDNADJ-1. In addition, in order to investigate the role of miR24/DJ-1 in rotenone-induced dopaminergic neuron injury, MN9D cells were treated with rotenone, miR-24 inhibitor, and si-DJ-1. MTT assay results revealed that rotenone reduced MN9D cell activity, while the transfection of miR24 inhibitor reversed the effect of rotenone (Figure 3F). After the transfection of si-DJ-1, the activity of MN9D cells was reduced again (Figure 3F).

\section{Interfering with miR-24 relieved nerve injury in PD rats}

To verify the mechanism of miR-24 on nerve injury, we compared the $\mathrm{NC}$ and miR-24 inhibitor groups. Compared with the NC group, the rats in the miR-24 inhibitor group exhibited a significant deterioration in motor performance and coordination (Figure 4A). In addition, miR-24 expression was downregulated and DJ-1 expression was upregulated in the brain tissues of rats in the miR-24 inhibitor group (Figure 4B, C). The LC3-II/LC3-I ratio and the protein expression of Beclin 1 were increased in the brain tissues of rats in the miR-24 inhibitor group (Figure 4D).

\section{Discussion}

This study reveals a significant increase in miR-24 expression in the brain tissues of PD rats and rotenone-induced dopaminergic neuronal cells. These results indicated that miR-24 might be related to the occurrence of PD. This is the first study on the role of miR-24 in PD.

MiRNAs have been reported to regulate various biological processes, including cell proliferation, apoptosis, and metastasis. Recently, miRNAs have been reported to regulate the development of PD. Kim et al. [19] showed that miR126 contributed to PD by downregulating IGF-1/PI3K/ AKT signaling. Li et al. [20] reported that overexpression of miR-221 promoted the viability and proliferation of 


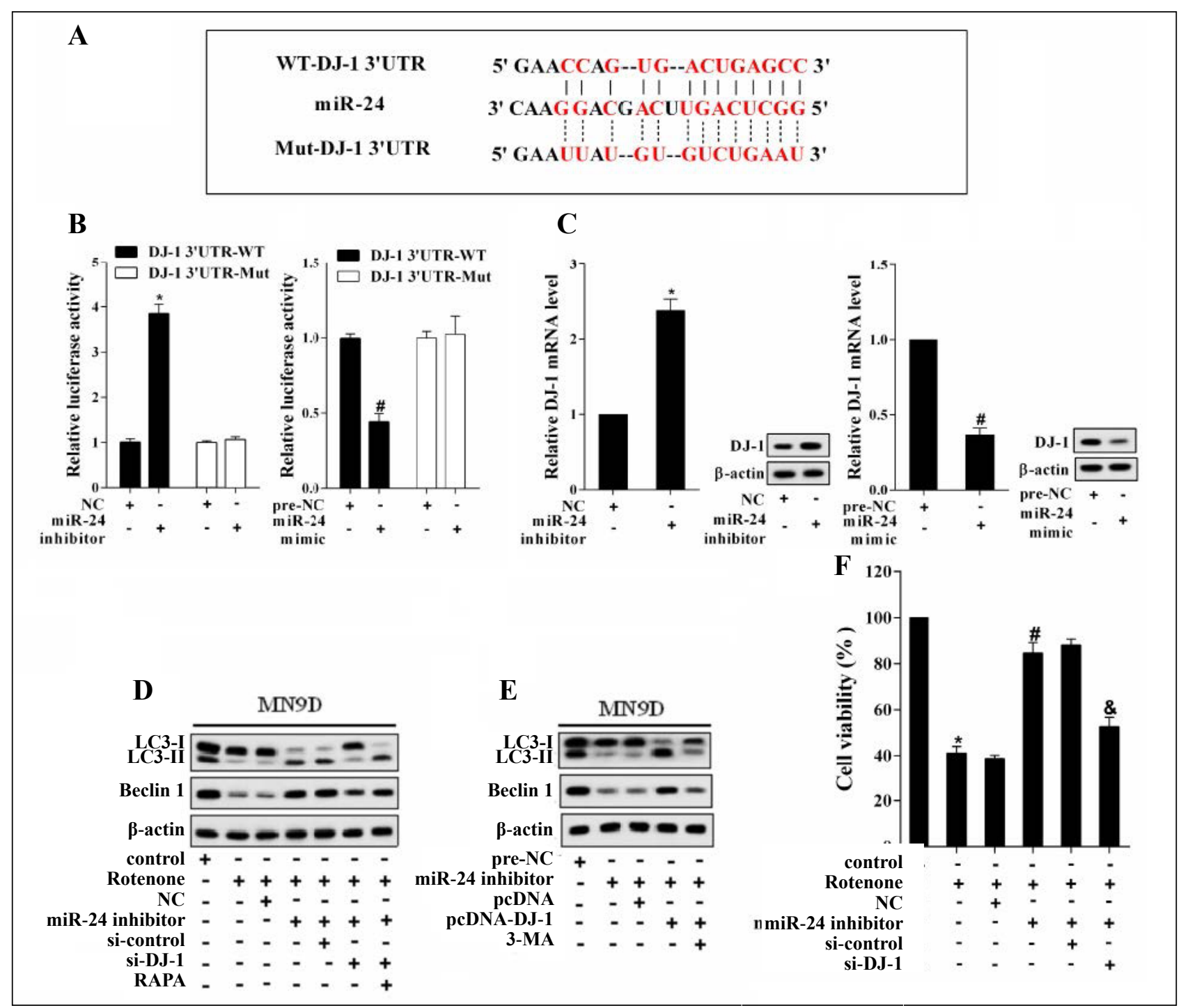

Figure 3. The role of miR-24 and DJ-1 in autophagy. (A) The binding sites between miR-24 and DJ-1 (TargetScan). (B) The relative luciferase activity. $* P<0.05$, vs NC. $\# P<0.05$, vs pre-NC. (C) DJ-1 expression in MN9D cells transfected with miR-24 inhibitor or mimic. $* P<0.05$, vs NC. \# $P<0.05$, vs pre-NC. (D) MN9D cells were divided into control, Rotenone, Rotenone + NC, Rotenone + miR-24 inhibitor, Rotenone + miR-24 inhibitor + si-control, Rotenone + miR-24 inhibitor + si-DJ-1, and Rotenone + miR-24 inhibitor + si-DJ-1 + RAPA group. The protein levels of LC3-I, LC3II, and Beclin 1 were measured by western blot. (E) MN9D cells were divided into pre-NC, miR-24 mimic, miR-24 mimic + pcDNA, miR-24 mimic + pcDNA-DJ-1, and miR-24 mimic + pcDNA-DJ-1 + 3-MA group. The protein levels of LC3-I, LC3-II, and Beclin 1 were measured by western blot. (F) MN9D cell activity. ${ }^{*} P<0.05$, vs control. $\# P<0.05$, vs Rotenone+NC. \& $P<0.05$, vs Rotenone+miR-24 inhibitor+si-control.

nerve cells in PD. In addition, miRNAs have been shown to be involved in autophagy in PD. For instance, upregulation of miR-124 was found to regulate impaired autophagy processes in PD by suppressing Bim expression [21], and miR-7 was found to promote autophagy in PD by reducing $\alpha$-synuclein levels [22]. Our study indicated that interfering with miR-24 alleviated rotenone-induced dopaminergic neuronal cell injury, and the effect of miR24 might be related to autophagy.

Autophagy is crucial in cell differentiation, proliferation, death, and response to environmental stress. It plays an important role in preventing certain diseases, such as cancer and neurodegenerative diseases, resisting infection by pathogenic microorganisms, and delaying aging, prolonging life span [23]. DJ-1 is an important protective protein, which plays a neuroprotective role in many reactions. For example, overexpression of DJ-1 was found to increase cell viability and decrease cell sensitivity to oxidative stress through the ERK1/2 signaling pathway [24]. In PD, it has been reported that DJ-1 reduces the neurotoxicity of rotenone on dopaminergic neurons by enhancing autophagy [25]. This is in line with the findings of our study. Moreover, our results showed that the role of DJ-1 in enhancing autophagy was related to miR-24. As miR24 negatively regulated DJ-1 expression, interfering with it enhanced autophagy by increasing DJ-1 expression and then relieved rotenone-induced dopaminergic neuronal cell injury. The PD rat model confirmed these findings. In conclusion, our in vitro and in vivo experiments revealed the role of miR-24/DJ-1 in autophagy in PD and demonstrated that interfering with miR-24 alleviated rotenone-induced dopaminergic neuron injury via enhancing 


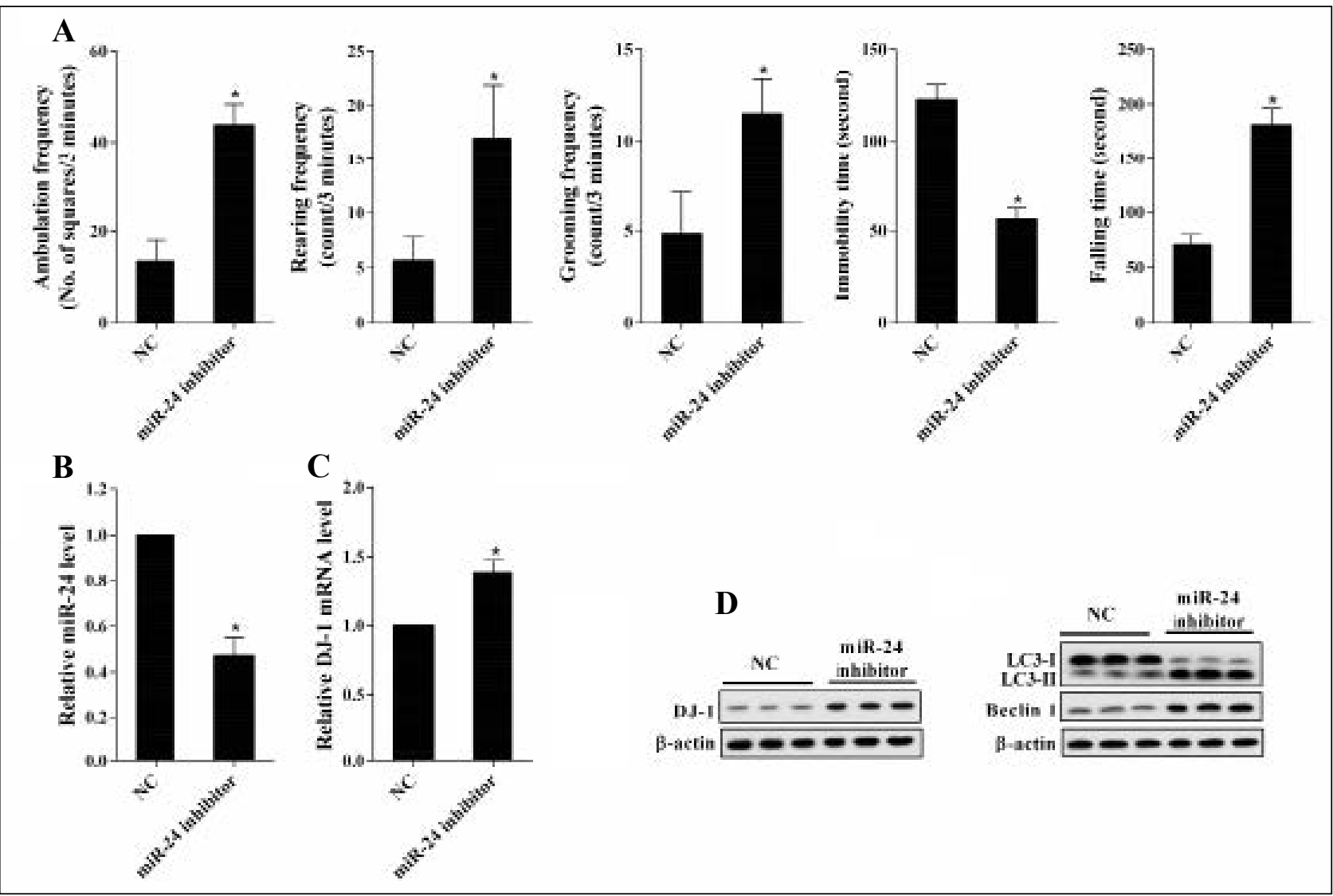

Figure 4. Interfering with miR-24 relieved nerve injury in PD rats. PD rats were divided into an NC group and a miR-24 inhibitor group of 6 rats each. (A) Open field test and rotarod test. * $P<0.05$, vs NC. (B) MiR-24 expression. ${ }^{*} P<0.05$, vs NC. (C) DJ-1 expression. $* P<0.05$, vs NC. (D) The protein levels of LC3-I, LC3-II, and Beclin 1.

autophagy by upregulating DJ-1, which had the potential to be a biomarker for the prevention and treatment of PD.

\section{Declarations}

Funding: This study was supported by the Natural science foundation of Inner Mongolia autonomous region (no. 2017MS0301).

Conflict of Interest: The authors declare that they have no conflict of interest.

\section{References}

1. Hirsch L, Jette N, Frolkis A, et al. The incidence of Parkinson's disease: a systematic review and meta-analysis. Neuroepidemiology, 2016, 46(4): 292-300.

2. Lashuel H A, Overk C R, Oueslati A, et al. The many faces of $\alpha$-synuclein: from structure and toxicity to therapeutic target. Nature Reviews Neuroscience, 2013, 14(1): 38.

3. Karabiyik C, Lee M J, Rubinsztein D C. Autophagy impairment in Parkinson's disease. Essays in biochemistry, 2017, 61(6): 711-720.

4. Moors T E, Hoozemans J J M, Ingrassia A, et al. Therapeutic potential of autophagy-enhancing agents in Par- kinson's disease. Molecular neurodegeneration, 2017, 12(1): 11.

5. Saito, Y. DJ-1 as a Biomarker of Parkinson's Disease. Adv Exp Med Biol, 2017, 1037: 149-171.

6. Choi D J, Eun J H, Kim B G, et al. AP arkinson's disease gene, DJ-1, repairs brain injury through $\mathrm{S}$ ox 9 stabilization and astrogliosis. Glia, 2018, 66(2): 445-458.

7. De Miranda B R, Rocha E M, Bai Q, et al. Astrocytespecific DJ-1 overexpression protects against rotenoneinduced neurotoxicity in a rat model of Parkinson's disease. Neurobiology of disease, 2018, 115: 101-114.

8. Xu C Y, Kang W Y, Chen Y M, et al. DJ-1 inhibits alphaSynuclein aggregation by regulating chaperone-mediated autophagy. Frontiers in aging neuroscience, 2017, 9: 308.

9. Zhang L, Lu Y, Zhang L. MiR-449b inhibits the migration and invasion of colorectal cancer cells through the negative regulation of MMP2. Clinical Surgery Research Communications, 2018, 2(3): 27-33.

10. Ma W, Li Y, Wang C, et al. Serum miR-221 serves as a biomarker for Parkinson's disease. Cell biochemistry and function, 2016, 34(7): 511-515.

11. Li D, Yang H, Ma J, et al. MicroRNA-30e regulates neuroinflammation in MPTP model of Parkinson's disease by targeting Nlrp3. Human cell, 2018, 31(2): 106-115.

12. Wen Z, Zhang J, Tang P, et al. Overexpression of miR185 inhibits autophagy and apoptosis of dopaminergic neu- 
rons by regulating the AMPK/mTOR signaling pathway in Parkinson's disease. Molecular medicine reports, 2018, 17(1): 131-137.

13. Kang H, Rho J G, Kim C, et al. The miR-24-3p/p130Cas: a novel axis regulating the migration and invasion of cancer cells. Scientific reports, 2017, 7: 44847.

14. Vallelunga A, Ragusa M, Di Mauro S, et al. Identification of circulating microRNAs for the differential diagnosis of Parkinson's disease and Multiple System Atrophy. Frontiers in cellular neuroscience, 2014, 8: 156.

15. Tong $X$, Wang $X$, Wang $C$, et al. Elevated levels of serum MiR-152 and miR-24 in uterine sarcoma: potential for inducing autophagy via SIRT1 and deacetylated LC3. British journal of biomedical science, 2018, 75(1): 7-12.

16. Barnum C J, Tansey M G. Neuroinflammation and nonmotor symptoms: the dark passenger of Parkinson's disease?. Current neurology and neuroscience reports, 2012, 12(4): 350-358.

17. Abdelkader N F, Safar M M, Salem H A. Ursodeoxycholic acid ameliorates apoptotic cascade in the rotenone model of Parkinson's disease: modulation of mitochondrial perturbations. Molecular neurobiology, 2016, 53(2): 810-817.

18. Gao H, Yang W, Qi Z, et al. DJ-1 protects dopaminergic neurons against rotenone-induced apoptosis by enhancing ERK-dependent mitophagy. Journal of molecular biology, 2012, 423(2): 232-248.

19. Kim W, Lee Y, McKenna N D, et al. miR-126 contributes to Parkinson's disease by dysregulating the insulin-like growth factor/phosphoinositide 3-kinase signaling. Neurobiology of aging, 2014, 35(7): 1712-1721.

20. Li L, Xu J, Wu M, et al. Protective role of microRNA-221 in Parkinson's disease. Bratislavske lekarske listy, 2018, 119(1): 22-27.

21. Wang H, Ye Y, Zhu Z, et al. MiR-124 Regulates Apoptosis and Autophagy Process in MPTP Model of P arkinson's Disease by Targeting to B im. Brain pathology, 2016, 26(2): 167-176.

22. Choi D C, Yoo M, Kabaria S, et al. MicroRNA-7 facilitates the degradation of alpha-synuclein and its aggregates by promoting autophagy. Neuroscience letters, 2018, 678: 118-123.

23. Levine B, Kroemer G. Autophagy in the pathogenesis of disease. Cell, 2008, 132(1): 27-42.

24. Gu L, Cui T, Fan C, et al. Involvement of ERK1/2 signaling pathway in DJ-1-induced neuroprotection against oxidative stress. Biochemical and biophysical research communications, 2009, 383(4): 469-474.

25. Hui-Feng L, Wei-Wei Y, Hua G, et al. DJ-1 alleviates rotenone-induced cell injuries via enhancing autophaty in MN9D cells. Basic \& Clinical Medicine, 2013, 33(6):648654.

Cite this article as: $\mathrm{Ge} \mathrm{B}, \mathrm{Wu} \mathrm{H}$, Shao $\mathrm{D}$, et al. Interfering with miR-24 alleviates rotenone-induced dopaminergic neuron injury via enhancing autophagy by upregulating DJ-1[J]. Aging Pathobiology and Therapeutics, 2019, 1(1): 17-24. 